\title{
Detrimental Effects of an Electronic Health Records System on Musculoskeletal Symptoms among Health Professionals
}

\author{
Alan Hedge ${ }^{1}$ and Tamara James ${ }^{2}$ \\ 1. Department of Design and Environmental Analysis, Cornell University, Ithaca NY 14853, USA. \\ 2. Community and Family Medicine, Occupational and Environmental Medicine, Duke University \\ Medical Center, Durham, NC 27710, USA.
}

\begin{abstract}
A survey of 204 health professionals (physicians, physicians' assistants and nurse practitioners) in private diagnostic clinics of a major healthcare system was conducted after the introduction of an electronic health records (EHR) system. Results showed considerable daily use of computers in various configurations and some $90 \%$ of respondents said the EHR had substantially increased their daily computer use. Less than half of the health professionals found the EHR easy to use. Almost half of the physicians said that use of the EHR reduced their face-to-face interactions with patients. Around two-thirds of respondents reported increased frequency of neck, shoulder and back discomfort and some 50\% reported an increased frequency of right wrist discomfort since introduction of the EHR. Results demonstrate the importance of incorporating ergonomic workstation designs and ergonomics education when an EHR is being implemented.
\end{abstract}

\section{INTRODUCTION}

An electronic medical record (EMR) is a digital version of a patient's medical history that includes complete information on diagnoses, medications, treatment plans, immunization dates, allergies, radiology images, and laboratory and test results. An electronic health record (EHR) goes beyond an EMR by also sharing this information with all other health care providers involved in the patient's care.

A 2013 study of office based physicians found that a large majority (78.4\%) now use some form of electronic health records (EHR) software (Hsiao \& Hing, 2014). The US Government has promoted the use of healthcare information technology (HIT) through legislation, such as the American Recovery and Reinvestment Act of 2009 (ARRA) which provided $\$ 20$ billion to fund the adoption of HIT by hospitals, and the Health Information Technology for Economic and Clinical Health (HITECH) Act of 2009, which authorized increased incentive payments to physician who use an EHR. The assumption behind these policies is that greater utilization of HIT will improve the clinical workflow, decrease medical errors and reduce costs. Indeed the "21st Century Roadmap for Advancing America's Health" report states that "If used in innovative ways, the estimated savings from HIT expansion could reach \$261 billion over 10 years." (Blumenthal \& Cortese, 2010).

Numerous studies have assessed the impact of HIT on healthcare providers. In a study of EMR use between 2006-7 by 2,625 physicians for 62,710 patient visits, results showed increases of $7.7 \%$ in any examination, $5.7 \%$ in any laboratory test, $4.9 \%$ in any health education, $7.1 \%$ fewer laboratory tests and $7.3 \%$ fewer radiology procedures during pre/postsurgery visits (Furukawa, 2011). A review of 154 studies of HIT implementation found positive results for the healthcare systems in $92 \%$ of studies (Buntin et al., 2011). A controlled pre-post, time and motion study of 129 doctors and nurses for 633.2 hours on four wards in a 400-bed hospital in Sydney, Australia, found that introduction of an EMR system resulted in reductions in prescribing error rates relative to the control wards, and it did not affect the time devoted to direct care or towards medication tasks (Westbrook et al., 2013). Doctors spent $19.7 \%(2 \mathrm{~h} / 10 \mathrm{~h}$ shift $)$ of their time on direct care and $7.4 \%$ (44.4 $\mathrm{min} / 10 \mathrm{~h} \mathrm{shift)}$ on medication tasks in the postperiod control ward, compared to $25.7 \%(2.6 \mathrm{~h} / 10 \mathrm{~h}$ shift; $\mathrm{p}=0.08)$ and $8.5 \%$ (51 $\mathrm{min} / 10 \mathrm{~h}$ shift; ns), respectively for intervention ward doctors. On the intervention ward doctors spent less time alone $(\mathrm{p}=0.0003)$ and more time with other doctors $(\mathrm{p}=0.003)$ and patients $(\mathrm{p}=0.009)$. Similar patterns were seen for control and intervention ward nurses in the postperiod (Westbrook et al., 2013). Analysis of data from 567 U.S. hospitals showed that HIT is associated with a swifter, more even patient flow, which results in improved revenues (Devaraj et al., 2013).

However, previous studies of HIT use among physicians have not always yielded such positive results. The impact of HIT on the quality and intensity of care delivered to Medicare inpatients was analyzed using data from 2.5 million inpatient admissions across 3,900 hospitals (Agha, 2014). Results showed that the adoption of HIT may improve patient health but may either increase or decrease medical expenditures, and that there was an initial $1.3 \%$ increase in billed charges with HIT but no evidence of cost savings, even five years after adoption (Agha, 2014).

A retrospective before/after analysis of 23 Emergency Departments (EDs) from a single management group that experienced ED EHR implementation and found no meaningful difference in 8 measures of operational performance (Ward et al., 2014).

The focus of almost all of the studies conducted to date has been on outcome measures for the healthcare system, such as measures of patient care or quantification of costs. Thus far there has been relatively little consideration of the health and well-being of the medical care personnel who are using HIT. However, previous studies of healthcare professionals using computers at poorly designed workstations have shown a substantial prevalence of musculoskeletal symptoms as well as 
other potentially detrimental changes in work practices (Hedge \& James, 2012a,b).

The present study reports on the impact of the introduction of a new EHR on the work and health of physicians, physicians' assistants and nurse practitioners working in private diagnostic clinics in a major healthcare system.

\section{Sample Profile}

\section{METHODS}

A self-selected sample of 146 physicians (MD), 24 physicians' assistants (PA) and 34 nurse practitioners (NP) working in outpatient Private Diagnostic Clinics (PDCs) in the Duke University School of Medicine and Health System in Durham, North Carolina voluntarily responded to an email invitation to participate in the study. The respondents comprised $41.5 \%$ of all PDC MD, PA and NP.

\section{Survey Instrument}

A web-based survey questionnaire was developed which comprised 30 questions (a total of 95 items) asking about demographic information, patterns of computer use at work and at home, the frequency of any musculoskeletal discomfort for 21 body segments (never, 1-2x/wk, 3-4x/wk, every day, several times/day) and how the discomfort frequency had changed (more frequent, no change or less frequent) since the introduction of the EHR (MaestroCare/Epic). The questionnaire had four sections: personal information (gender, age, length of working in years/months, and clinical location of work); use of different computer technology (desktop, laptop, table/iPad, smartphone) both at work and at home, including questions on the frequency of use and adjustment to various items of computer equipment, the kind of computer work undertaken, ease of use of the new EMR and typing proficiency, whether a computer related injury had been experienced and whether any interventions have been undertaken to correct the situation. Finally, there was an open ended comments section.

\section{Procedure}

The survey was electronically administered some three months after introduction of the new EHR system. An email invitation to participate in the survey was sent to PDC MD, PA and NP, and two-weeks later a follow-up email invitation was sent. The survey instrument and procedure were approved by the IRBs at Cornell University and Duke University School of Medicine.

\section{Data Analysis}

All data were analyzed using a multivariate statistical package (SPSS v22). Written comments were content analyzed.

\section{RESULTS} Table 1.

The profile of the survey sample by job is shown in

There was intensive use of the EHR, especially by the PA, NP (Figure 1). Since the introduction of the EHR a large majority of respondents reported much more or more clinic computer use $(\mathrm{MD}=86.2 \% ; \mathrm{PA}=88.0 \% ; \mathrm{NP}=91.1 \%)$.

Table 1: Profile of the survey respondents by job

\begin{tabular}{|l|c|c|c|}
\hline & MD & PA & NP \\
\hline $\mathrm{N}$ & 146 & 24 & 34 \\
\hline \% female & $41.0 \%$ & $69.6 \%$ & $94.1 \%$ \\
\hline$>=50$ years & $46.5 \%$ & $48.0 \%$ & $29.4 \%$ \\
\hline 5+ clinic years & $72.6 \%$ & $64.0 \%$ & $52.9 \%$ \\
\hline 8+ hours of clinic computer use/day & $46.6 \%$ & $52.0 \%$ & $35.3 \%$ \\
\hline Typing skill (touch typist) & $72.4 \%$ & $60.0 \%$ & $87.9 \%$ \\
\hline $\begin{array}{l}\text { Daily computer work (equal } \\
\text { keyboard/mouse use) }\end{array}$ & $75.9 \%$ & $88.0 \%$ & $84.8 \%$ \\
\hline
\end{tabular}

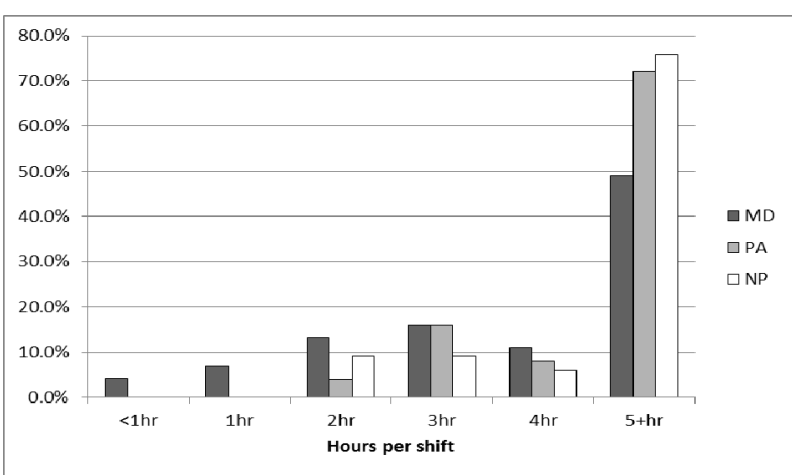

Figure 1: Hours per day using the EHR by job

The daily hours in face-to-face contact with patients for each job are shown in Figure 2.

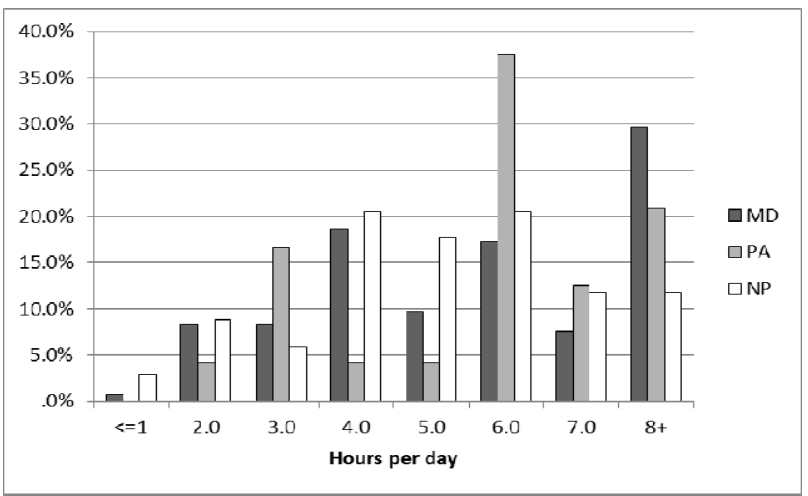

Figure 2. Hours per day in face-to-face interactions with patients by job

Around half of the MD (47.6\%) and NP (53.0\%) and a quarter of the PA $(24.0 \%)$ reported much less or less face-toface time with patients since the introduction of the EHR (Figure 3).

A large majority of the respondents reported using a desktop computer either daily or weekly (Table 2). Only around one third of MD reported regularly adjusting the position of their keyboard or monitor, whereas such adjustments were reported by a higher percentage of PA and NP. The use of voice recognition software was reported by 45.9\% MD, 62.5\% PA and 28.1\% NP. Problems with using the VR software were reported by $82.8 \%$ of MD users, $44.4 \%$ of PA users and $55.6 \%$ of NP users. 


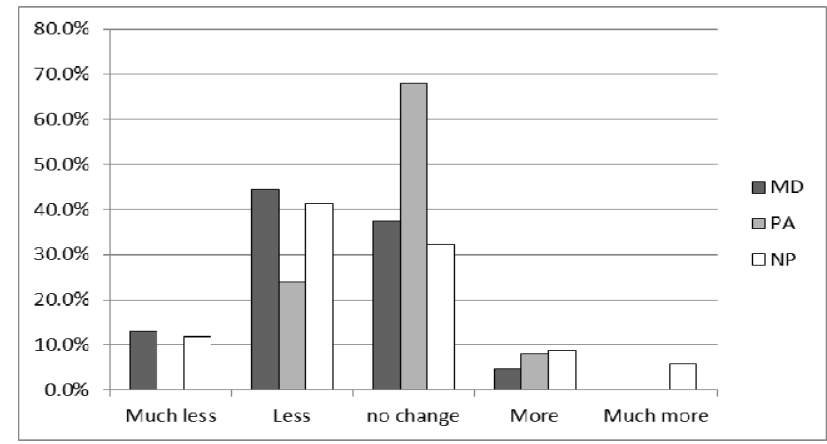

Figure 3: Change in face-to-face time interacting with patients post EHR by job

Around half of the respondents made at least weekly use of a wall mounted computer and two thirds of the PA made regular adjustments to the keyboard and screen position to this whereas only around one third of MD and NP did this. Only a minority of respondents used a computer on a mobile cart.

Table 2. Computer use by the survey respondents by job

\begin{tabular}{|l|l|l|l|}
\hline & \multicolumn{1}{|c|}{ MD } & \multicolumn{1}{|c|}{ PA } & \multicolumn{1}{|c|}{ NP } \\
\hline Desktop computer use (weekly/daily) & $83.6 \%$ & $88.0 \%$ & $78.8 \%$ \\
\hline Desktop keyboard adjustment & $33.6 \%$ & $68.0 \%$ & $49.9 \%$ \\
\hline Desktop monitor adjustment & $28.1 \%$ & $36.0 \%$ & $37.5 \%$ \\
\hline Wall Mount computer use (weekly/daily) & $47.0 \%$ & $59.1 \%$ & $46.7 \%$ \\
\hline Wall mount keyboard adjustment & $35.0 \%$ & $65.0 \%$ & $27.3 \%$ \\
\hline Wall mount monitor adjustment & $40.0 \%$ & $65.0 \%$ & $40.9 \%$ \\
\hline Computer cart use & $19.4 \%$ & $0 \%$ & $11.6 \%$ \\
\hline Computer cart keyboard adjustment & $27.0 \%$ & $0 \%$ & $18.7 \%$ \\
\hline Computer cart monitor adjustment & $25.0 \%$ & $8.3 \%$ & $18.7 \%$ \\
\hline Computer cart sufficient mouse space & $25.0 \%$ & $16.7 \%$ & $36.4 \%$ \\
\hline
\end{tabular}

Only a small minority of respondents said that the new EHR system was very easy to use (Figure 4), whereas around half of respondents said that this was either very or fairly difficult to use ( $\mathrm{MD}=57.8 \%$; $\mathrm{PA}=48 \%$; $\mathrm{NP}=51.5 \%$ ).

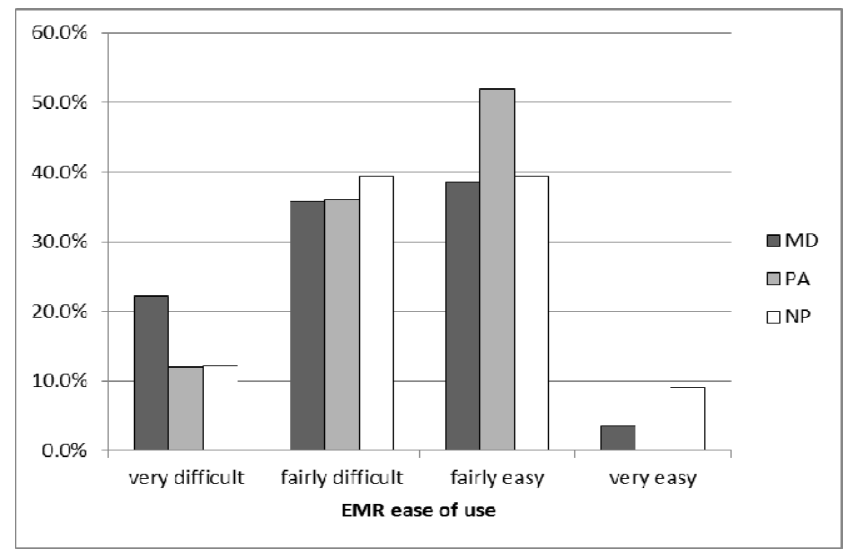

Figure 4. EHR ease of use by job

A large majority of respondents reported that they mostly sit to work at a computer (MD=86.9\%; $\mathrm{PA}=84.0 \% ; \mathrm{NP}=97.0$ ).
A large majority of the respondents reported that their workplace layout hinders the quality of patient care provided (Figure 5).

Reports of musculoskeletal discomfort experienced at least weekly showed a very high prevalence of neck, shoulder and upper back symptoms affecting at least three quarters of respondents, and especially the PA (Figure 6). Lower back and right wrist problems affected some $50-60 \%$ of respondents.

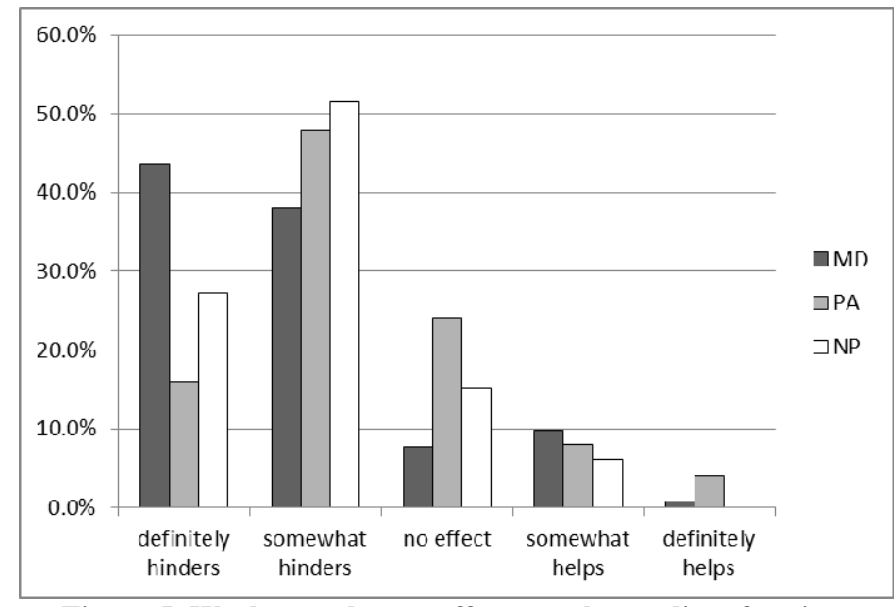

Figure 5. Workspace layout effects on the quality of patient interactions post EHR by job

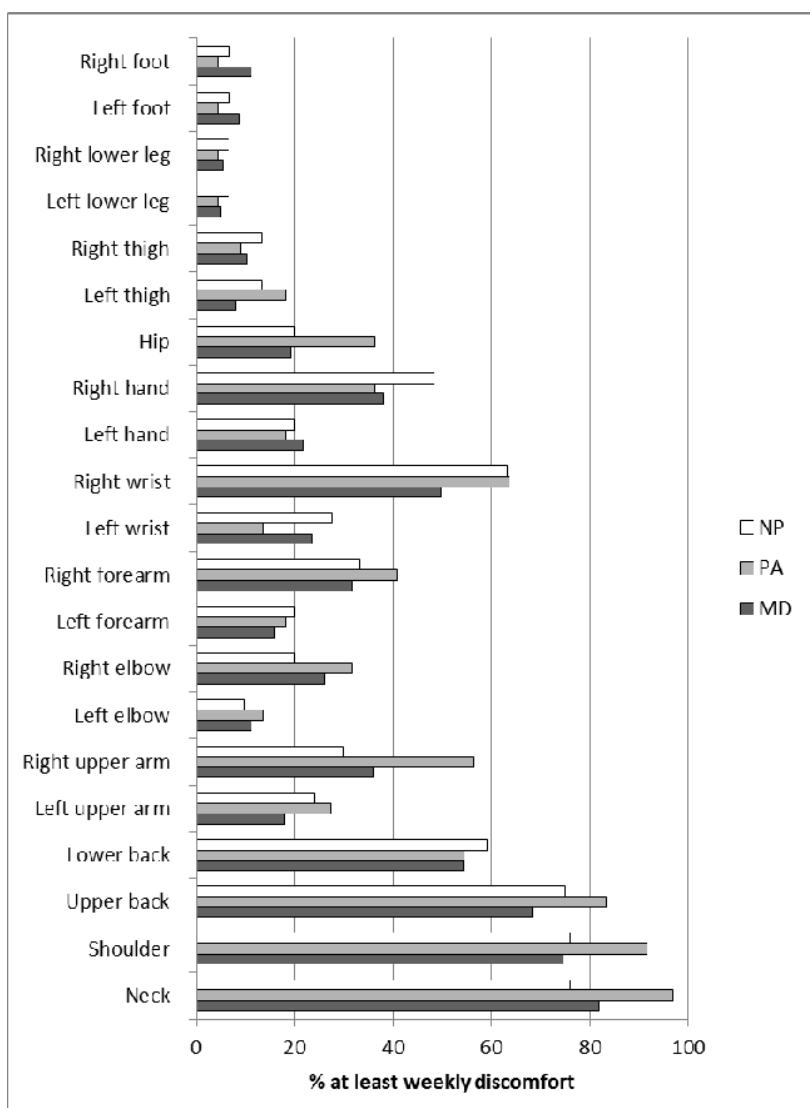

Figure 6. Prevalence of at least weekly musculoskeletal discomfort by job

When asked how their experience of musculoskeletal discomfort had been affected by the introduction of the new 
EHR a substantial majority of respondents indicated that their discomfort had become more frequent, and this was especially true for the neck, shoulders, upper back and right wrist (Figure 7).

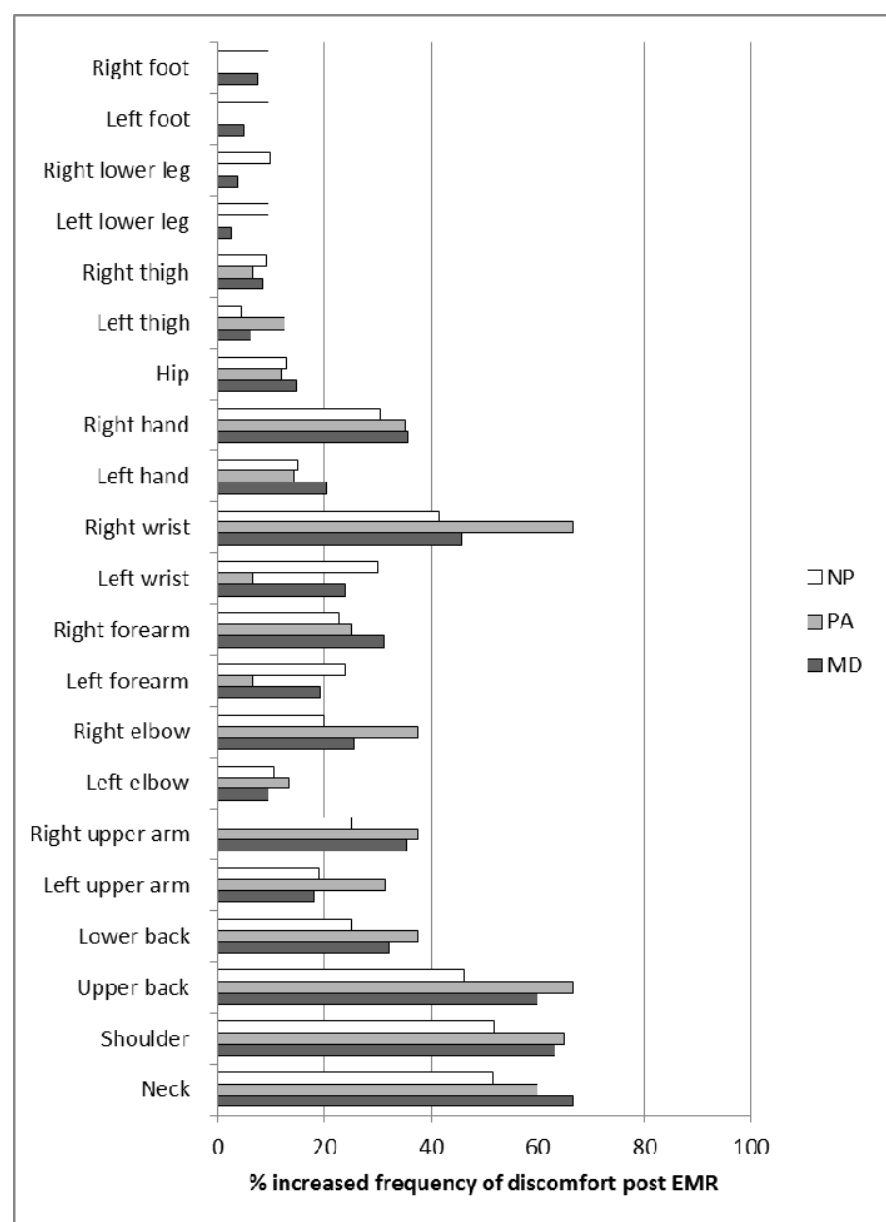

Figure 7. Change in the prevalence of musculoskeletal discomfort by job after introduction of the EHR

When asked whether they had ever experienced a computer-related injury at work, 16 MDs, 3 PAs and 6 NPs reported various neck and upper limb injuries.

Finally, $53.9 \%$ of respondents wrote comments and all of these were negative and critical of the EHR, its adverse impact on productivity and the poor design of the workplace to support more intensive computer use.

\section{DISCUSSION}

The use of an EHR is being heavily promoted by legislation based on the assumption that this software will speed the clinical workflow, reduce medical errors, improve the patient experience, and reduce costs. However, to date little attention has been paid to the changes in workload for the healthcare professionals or the potential adverse health issues associated with workplaces that are poorly designed to support intensive computer use. This survey has revealed a very high prevalence of upper body musculoskeletal discomfort and it has shown that this has worsened since the introduction of the EHR. Moreover, the survey has shown that EHR use in poorly designed workplaces can adversely affect the quality of patient interactions. For example, numerous comments indicated that in many of the workspaces the position of the computer meant that a choice had to be made between facing the patient or facing the computer, and this negatively affected the quality of interaction with the patient.

The participating healthcare system invested a considerable sum of money in the software and associated hardware, but corresponding resources were not allocated to modify clinic settings by ensuring ergonomic workstation furniture and reorganizing room layouts for optimal use of this EHR.

Previous studies of healthcare professionals in this PDC system had documented widespread musculoskeletal symptoms and deficiencies in workplace layouts (Hedge \& James, 2012a,b). However, introduction of the new EHR system requires even more intensive computer use and in the time since its introduction this has resulted in an even higher prevalence and frequency of musculoskeletal discomfort. The fact that not one positive comment was received about the EHR, whereas over half of respondents wrote extensive negative comments about the impact of the EHR on their health, work productivity and workplace, highlights the need to consider the ergonomic design of computer workspaces to better accommodate intensive computer use, as well as the impact of an EHR on the work content and productivity of healthcare professionals. The high levels of musculoskeletal discomfort associated with computer use reported in the present study confirm and exceed earlier findings (Hedge \& James, 2012a,b) and these levels are considerably greater than those often seen in commercial office workplaces (e.g. Hedge et al., 2011). It should be of great concern that over $60 \%$ of respondents reported that their musculoskeletal discomfort was more frequent after introduction of the new EHR.

While the survey addressed health concerns it did not systematically collect data on the impact of the EHR on work performance. However, numerous written comments noted that the EHR required significantly more computer time to work on notes and that this system added to the daily work time and workload rather than reducing this. As one physician noted, "the result of the EHR is less physician patient interaction and a degradation of quality of care". Other physicians noted that the EHR was not an intuitive system and that it was outdated software that requires too many clicks and is too time consuming. Indeed, in the words of other physicians this particular EHR is "very poorly written with terrible ergonomics and does not adhere to any Windows conventions (i.e., mouse use, right click for properties, any help, location of menus, etc.), and this EHR "has destroyed efficient workflow and is a horrible tool for communication". Negative comments and perceptions are to be expected when large scale changes such as new EHR systems are implemented. However, such comments raise serious questions about the process for selecting modern, intuitive and effective EHR software, and they highlight the importance of evaluating the ergonomic design and interaction of both the software interface and the supporting workspace environment. 
In our previous publications (Hedge \& James, 2012a,b) we noted the following. "The clinics that participated in this survey will be migrating to a new electronic medical records software system that will require everyone to use computers for direct entry. If the clinics do not also adopt the use of voice recognition software and require only keyboard and mouse input it is highly probable that the prevalence, frequency and severity of musculoskeletal symptoms will increase." It seems that our predictions have become a reality for many of the providers. In our view the consequences of not considering ergonomics in the selection and implementation of an EHR can be catastrophic for the healthcare professionals. One physician noted that "Hand surgeons should be jumping for joy. The number of newly diagnosed hand/wrist repetitive motion injuries is going to sky-rocket with the volume of typing and clicking necessary to navigate a patient encounter in EHR."

The expansion of information technology in healthcare is imminent. The reality for healthcare professionals is that computer use is a substantial part of their daily work and this will increase rather than decrease in the future. Healthcare professionals now use computing devices at work and at home, and such technology increasingly will become more mobile.

For those healthcare systems that have already made large investments in HIT systems, it is not likely the ergonomic design of the software will change significantly in the immediate future. However, they can still optimize clinical workflow as well as prevent injury through improving the design of the supporting physical environments. Moreover, the effective training of healthcare professionals in good ergonomics practices also is widely neglected and would help with injury prevention.

Health systems that have not yet invested in HIT should carefully evaluate, select, and implement effective and efficient HIT systems that utilize good ergonomic design of the software (such as reducing mouse clicks and including good voice recognition software and training) in addition to ergonomic design of the clinical workplace while concurrently optimizing workflow for better patient interactions and greater quality of care. Lastly, to truly facilitate work performance efficiency and safety, EHR software developers must do a better job of including end users in the design of their product.

The importance of the ergonomic design of clinical workplaces to protect medical personnel against musculoskeletal injury risks and promote effective working is emphasized by the findings of this survey.

\section{ACKNOWLEDGEMENTS}

The authors would like to acknowledge Paul Newman, Executive Director of the Private Diagnostic Clinics for providing email access to the PDC members and to his assistant, Michelle Taylor for overseeing distribution of the email invitations.

\section{REFERENCES}

Agha, L. 2014. The Effects of Health Information Technology on the Costs and Quality of Medical Care. Journal of Health Economics, 34, March:19-30

Blumenthal, S.J. \& Cortese, D.A. 2010. A 21st Century Roadmap For Advancing America's Health: The Path From Peril To Progress. Center for the Study of the Presidency and Congress, Washington D.C.

Buntin, M.B., Matthew F. Burke, M.F., Hoaglin, M.C. \& Blumenthal, D. 2011. The benefits of health information technology: a review of the recent literature shows predominantly positive results. Health Affairs, 30(3):464-471.

Devaraj, S., Ow, T. T., \& Kohli, R. 2013. Examining the impact of information technology and patient flow on healthcare performance: A Theory of Swift and Even Flow (TSEF) perspective. Journal of Operations Management, 31(4), 181-192.

Furukawa, M. F. 2011. Electronic medical records and efficiency and productivity during office visits. American Journal of Managed Care, 17(4), 298-303.

Hedge, A. James, T. \& Pavlovic, S. 2011. Ergonomics Concerns and the Impact of Healthcare IT, International Journal of Industrial Ergonomics, 41 (4), 345-351.

Hedge, A. and James, T. 2012a. Ergonomic issues of computer use in a major healthcare system, In Proceedings of the 4th international Conference on Applied Human Factors \& Ergonomics 2012, July 21-25, San Francisco, C, 2630-2639.

Hedge, A. and James, T. 2012b. Gender Effects on Musculoskeletal Symptoms among Physician Computer Users in Outpatient Diagnostic Clinics, In Proceedings of the Human Factors and Ergonomics Society 56th Annual Meeting, October 22-26, Boston, MA. 887-891.

Hsiao C.J, Hing E. 2014. Use and characteristics of electronic health record systems among office-based physician practices: United States, 2001-2013. NCHS data brief, no 143. Hyattsville MD: National Center for Health Statistics.

Ward, M. J., Landman, A. B., Case, K., Berthelot, J., Pilgrim, R. L., \& Pines, J. M. 2014. The Effect of Electronic Health Record Implementation on Community Emergency Department Operational Measures of Performance. Annals of Emergency Medicine, 63(6):723-30.

Westbrook, J. I., Li, L., Georgiou, A., Paoloni, R., \& Cullen, J. 2013. Impact of an electronic medication management system on hospital doctors' and nurses' work: a controlled pre-post, time and motion study. Journal of the American Medical Informatics Association, 20 (6):1150-1158. 